\title{
Prognostic Value of Standard Coronary Computed Tomography Angiography Reporting System (CAD-RADS)
}

\author{
Sedat Altay ${ }^{1, \odot}$
}

Address for correspondence Sedat Altay, MD, Department of Radiology, Izmir Katip Celebi University Ataturk Research and Training Hospital, Polat Caddesi, 35140 Izmir, Turkey (e-mail: sedataltay@yahoo.com).

\begin{abstract}
Keywords

- CAD-RADS

- computed tomography angiography

- coronary artery disease

- prognosis

Aims This study evaluated the clinical prospects of Coronary Artery DiseaseReporting and Data System (CAD-RADS) scoring in coronary computed tomography angiography (CTA). The aim of the study was to determine the guidance value of CAD-RADS scoring in patient follow-up after CTA.

Methods and Materials Reports of cases reported between 2010 and 2013 were reevaluated with CAD-RADS scoring. Clinical risk analysis was performed with initial forms of anamnesis. Clinical follow-up was performed on 7 to 10 years (mean: 8 years, 4 months) hospital records. Univariate and multivariate Cox modeling was performed with Kaplan-Meier method to define the relationship between clinical (age, gender, diabetes mellitus, hypertension, smoking, family history) and CAD-RADS variables, and for risk analysis based on these causes. Cox proportional-hazards analysis results were presented as a hazard ratio with a 95\% confidence interval. CAD-RADS scores were evaluated as meaningful determinants of univariate and multivariate Cox proportional survival analysis.

Results Totally, 359 cases were evaluated in the study. Severe coronary pathology development rate was observed as CAD-RADS 0to 1\%, CAD-RADS 1 to 3\%, CAD-RADS 2 to $4 \%$, CAD-RADS 3 to $9 \%$, CAD-RADS 4 A to $21 \%, 4 B$ to $25 \%$, CAD-RADS 5 to $50 \%$. There were no coronary artery deaths in CAD-RADS 1,2,3 cases in 10 years of follow-up. Two cases with CAD-RADS 4 A score, three cases with $4 \mathrm{~B}$ score, and four patients with CAD-RADS 5 had a history of death as a result of coronary disease.

Conclusions The cases with a high risk of side effects with CAD-RADS scores were clearly shown. CAD-RADS score accurately identifies risks in postimaging follow-up and is a reliable reporting system in the required treatment planning.
\end{abstract}

\section{Introduction}

Cardiovascular diseases are the highest cause of mortality and morbidity in Western countries. Coronary computed tomography angiography (CTA) is a widely used diagnostic noninvasive imaging method for detecting coronary artery

published online May 31, 2021
DOI https://doi.org/

$10.1055 / \mathrm{s}-0041-1729128$ ISSN 0971-3026 diseases (CAD) due to a short examination time. Invasive coronary angiography (ICA) is the golden standard imaging method in the diagnosis of CAD (1). Due to the widespread and increasing use of CTA, a standard reporting method has been mandatory. The Coronary Artery Disease-Reporting and Data System (CAD-RADS) classification system is used

\footnotetext{
(c) 2021. Indian Radiological Association

This is an open access article published by Thieme under the terms of the Creative Commons Attribution-NonDerivative-NonCommercial-License, permitting copying and reproduction so long as the original work is given appropriate credit. Contents may not be used for commercial purposes, or adapted, remixed, transformed or built upon. (https://creativecommons.org/licenses/by-nc-nd/4.0/).

Thieme Medical and Scientific Publishers Private Ltd. A-12, Second Floor, Sector -2, NOIDA -201301, India
} 
as an international standard in CTA reporting (- Table 1). ${ }^{1,2}$ CAD-RADS standards have been beneficial for the development of a common language between radiologists and clinicians.

Studies on the relationship between survival and CTA findings in coronary patients have been conducted. ${ }^{3}$ In these studies, CAD was described as absent, mild, and severe, according to the state of stenosis. The widespread use of CAD-RADS classification does not have a very long history. There are few and short-term follow-up studies on the relationship between CAD-RADS classification and survival in the literature in the English language. The longest follow-up duration is 5 years, a short time to assess coronary pathologies. ${ }^{4}$ This study has a long-term follow-up.

The clinical results of reporting with CAD-RADS scores are examined with long-term studies. ${ }^{1,2,4}$ Many countries should work on this issue to develop their health policies in $\mathrm{CAD}$ cases. The main objective of this study is to examine the guiding potential of CAD-RADS scores in post-CTA follow-up and treatment.

\section{Methods}

Approval was obtained from the ethics committee of our hospital for the study. This study was designed as a multi-cantered, retrospective study.

All examinations were performed using 64 slices of Toshiba Multislice Aquilion systems (Toshiba Medical Systems, Tokyo, Japan). If the heart rate was 70 beats/min, additional oral $\beta$-blockers (metoprolol, $50 \mathrm{mg}$, single dose, 1 hour before scan) were provided if tolerated. First, a coronary calcium scan was performed before prospectively triggered MSCT angiography. Images for calcium scoring were acquired by a noncontract-enhanced scan. With the same parameters for the 64-section MSCT was Collimation $4 \times 3.0 \mathrm{~mm}$, slide rotation time 350 milliseconds, tube voltage and tube current $120 \mathrm{kV}$ and $200 \mathrm{mAs}$, respectively. The temporal window was set at 75\% after the R-wave for electrocardiogram-triggered prospective reconstruction. All images were reconstructed at the Vitrea (Vital, a Toshiba Medical Systems Group company, Minnesota, United States) workstation. Nonionic contrast material was administered in the antecubital vein 70 to $95 \mathrm{~mL}$. It was a flow rate of $4.5 \mathrm{ml} / \mathrm{s}$ (Iomeprol, Imeron 350, Bracco Altana Pharma Gmbh, Konstanz, Germany). Automated detection of peak enhancement $120 \mathrm{HU}$ in the aortic root was used for the timing of the scan. İmages analyzed with a commercially available software package, Vitrea.

Medical records were examined for 565 patients who were reported as standard between January 2010 and December 2013. A total of 565 CTA reports were retrospectively reviewed. The cases were rereported by CAD-RADS standards according to the stenosis percentages and pathologies defined in the reports. The patients were grouped according to the CAD-RADS value. ${ }^{5}$ The reporting was done between 2010 and 2013 by a radiologist who had 4 years of experience in reporting cardiac CT. Information about the patient was obtained with the clinical history described in the first admission and detailed consent forms signed by the patients before coronary CTA. imaging. Patients who had invasive cardiac angiography after CTA but not undergoing vascular treatment were included in the study. Hospital records were examined using an ID number. The cases were evaluated with 7 to 10 years of clinical follow-up information. CTA imaging reports were not evaluated again in the follow-up.

Totally, 359 patients who were reported in the radiology clinic between 2010 and 2013 according to the Society of Cardiovascular Computed Tomography guidelines were reported again with CAD-RADS scoring (-Table 1). In cases, stent and vulnerable plaque were not evaluated in CAD-RADS scoring.

\section{Statistical Method}

SPSS 24 (IBM) program was used for statistical analysis. A comparison between CAD-RADS scores was made using the chi-squared test for variables that can be categorized with baseline properties and variance analysis for continuous variables. We predicted survival rate without coronary pathology using Kaplan-Meier method. Major adverse cardiac events (MACE) were included in the statistics when they occurred after CTA. Univariate and multivariate Cox proportional hazards survival models are calculated by reporting the hazard ratio with a 95\% confidence interval. Univariate Cox proportional hazard analysis was shown. Variables such as age, gender, hypertension, diabetes mellitus, smoking, and family history were included in the Cox models for multivariate. Since coronary medical treatment was not followed prospectively, it was not included in the analysis. For all analyses using SPSS, the $p$-value $<0.05$ was considered statistically significant.

\section{Results}

For this study, 565 patients who underwent CTA between 2010 and 2013 and whose follow-up information was available in the hospital records were examined. Twenty-five patients who were given stenosis information in the previous report but did not have a stenosis percentage were excluded. The patients monitored for bypass and stent control (55) were excluded from the evaluation. Patients with insufficient records at first admission to the hospital (58 cases) were excluded from the study. A total of 28 patients who underwent coronary surgery after CTA examination and 129 patients without hospital follow-up for 3 years or longer were excluded. Twenty-four cases with CAD-RADS $1,2,3$ scoring without adequate coronary anamnesis were excluded among the cases who were admitted to different outpatient clinics in the same institution. The physicians of 22 cases with high CAD-RADS scores and insufficient hospital records were consulted. Despite all, six cases without sufficient clinical information were excluded from the study. During the follow-up, patients who developed noncoronary life-threatening pathology and had a history of operation were excluded from the study. Patients who died of noncoronary pathology were excluded from the evaluation. Of the 359 cases that could be examined, 164 were female (mean: 52 years old) and 195 were male (mean: 56 years old). 
Table 1 Case processing summary: Kaplan-Meier cumulative incidence uneventful table. Cumulative surveys were 98.9 for CAD-RADS and $55.6 \%$ for CAD-RADS

\begin{tabular}{|l|l|l|l|l|}
\hline CAD-RADS & $n$ & Number of events & \multicolumn{2}{l|}{ Censored } \\
\cline { 3 - 6 } & & & $n$ & \multicolumn{2}{l|}{} \\
\hline 0 & 91 & 1 & 90 & $98.9 \%$ \\
\hline 1 & 83 & 3 & 80 & $96.4 \%$ \\
\hline 2 & 75 & 3 & 72 & $96.0 \%$ \\
\hline 3 & 69 & 7 & 62 & $89.9 \%$ \\
\hline $4-a$ & 19 & 4 & 15 & $78.9 \%$ \\
\hline $4-b$ & 13 & 3 & 10 & $76.9 \%$ \\
\hline 5 & 9 & 4 & 5 & $55.6 \%$ \\
\hline Overall & 359 & 25 & 334 & $93.0 \%$ \\
\hline
\end{tabular}

Abbreviation: CAD-RADS, Coronary Artery Disease-Reporting and Data System.

When the previous reports of the cases were reevaluated, 91 cases (25\%) were classified as CAD-RADS 0, 83 cases (23\%) as CAD-RADS 1, 75 cases (20\%) as CAD-RADS 2, 69 cases (19\%) as CAD-RADS 3, 19 cases (5\%) as CAD-RADS 4A, 13 cases (13\%) as CAD-RADS 4B, and 9 cases (2\%) were reclassified as CAD-RADS 5. The cases were revised in according to CAD-RADS values based on age, gender, hypertension, diabetes mellitus history, smoking status, and family history of cardiac death. All cases were evaluated based on the first admission records. During the follow-up of the cases, newly diagnosed hypertension and diabetes mellitus, and altered smoking status were not evaluated. MACE observed in the cases were evaluated until January 2020.

The mean follow-up was 8 years and 4 months, the longest was 9 years and 8 months, and the shortest was 6 years and 8 months. During the follow-up, MACE myocardial infarction, sudden cardiac death, bypass surgery, other cardiac surgeries, or stent administration were recorded. Cases reported as CAD-RADS 4A, 4B, and 5 and followed by medical treatment without surgical treatment were also evaluated.

ICA was not applied to any of the patients in the CAD-RADS 0 and 1 group. ICA was performed in CAD-RADS 2 (5\%) and CAD-RADS 3 cases (12\%). In all cases, ICA was applied in the first year after CTA. ICA was applied to CAD-RADS 4A (50\%) and $4 \mathrm{~B}(55 \%)$ and CAD-RADS 5 (80\%) cases. About $80 \%$ of this examination was done in the first 6 months. Since patients with a history of coronary surgery were not included in the study, none of our patients had surgery.

As a result, the risk of developing MACE in follow-up increased in cases with increased CAD-RADS scores (-Table 2). MACE was observed in one of the CAD-RADS 0 cases (2\%). MACE was observed in three cases with a CAD-RADS score of 1. MACE was observed in three and seven cases with CAD-RADS scores of 2 and 3, respectively (4 and 9\%). MACE was detected in four cases with CAD-RADS 4A findings (mean: 3 years and 2 months), three patients with CAD-RADS 4B (mean: 3 years and 4 months), and four patients with CAD-RADS 5 classification (mean: 2 years and 5 months). Of the cases in whom MACE was defined, two cases with CAD-RADS 4A, two cases with CAD-RADS 4B, and three cases (mean: 2 years) with CAD-RADS 5 died from coronary causes. There was death from coronary causes in CAD-RADS 1,2,3 cases in 10 years.

Twenty-five MACE and seven coronary deaths were seen in the entire study group. Ten years of cumulative survival was noted between 1\% for CAD-RADS 5 and $50 \%$ for CAD-RADS 0 ( - Fig. 1 ) and ( - Table 3 ). In the multivariate Cox model, CAD-RADS scores were highly associated with MACE risk. Hazard ratios were calculated 1.31 for CAD-RADS 1 $(p=0.0012)$ and 7.12 for CAD-RADS $5(p=0.0004)(-$ Table 4$)$. Kaplan-Meier -Overall MACE incidence Comparisons. $\mathrm{P}<0.005$ was found significant. CAD-RADS prognostic performance was estimated by the receiver operating characteristic curve, and it was found similar to the Duke CAD index (0.7073) as $p=0.6946$.

In the multivariate Cox model, the CAD-RADS scores of 4 and 5 were strongly associated with a high risk of death or myocardial infarction ( - Fig. 2).

\section{Discussion}

This study examines the impact of CAD-RADS standard reporting on 10 years of the survey. The main result of the study is that the risk of developing CAD and the risk of coronary death is increased in patients with high CAD-RADS levels following the statistical exclusion of other factors. MACE were considered as myocardial infarction, sudden cardiac death, bypass surgery, other cardiac surgeries, or stent implementation., ${ }^{4,6-10}$ Mortality rate due to coronary pathology is high in a 10 -year follow-up, especially at CAD-RADS 4 and 5 levels. In cases with CAD-RADS 0, 1, and 2, the incidence of MACE is not different compared with the normal population. ${ }^{3}$ This study will prevent overtreatment for CAD in patients with CAD-RADS scoring and provide evidence-based foresight for invasive procedures. Long-term severe MACE development in cases of high CAD-RADS is an indication for treatment.

Clinical monitoring and treatment planning of coronary CTA patients are important. ${ }^{8,11}$ With its increasing use in reporting, CAD-RADS guideline is gaining more and more importance daily (5). A reliable standardization of radiological reports is helpful for the doctor in making medical treatment or ICA decision. ${ }^{11,12}$ In our study, the high level of 
Table 1 CAD-RADS classification system and further cardiac investigation

\begin{tabular}{|l|l|l|l|}
\hline Classification & Maximal stenosis & Interpretation & Further cardiac investigation \\
\hline CAD-RADS 0 & $0 \%$ & No CAD & None \\
\hline CAD-RADS 1 & $1-24 \%$ & Minimal nonobstructive & None \\
\hline CAD-RADS 2 & $25-49 \%$ & Mild nonobstructive & None \\
\hline CAD-RADS 3 & $50-69 \%$ & Moderate stenosis & Consider functional assessment \\
\hline CAD-RADS 4 A & $70-99 \%$ & Severe stenosis & $\begin{array}{l}\text { Consider ICA or functional } \\
\text { assessment }\end{array}$ \\
\hline CAD-RADS 4 B & Left main $>50 \%$ or 3-vessel $\geq 70 \%$ & Severe stenosis & ICA recommended \\
\hline CAD-RADS 5 & $100 \%$ & Total coronary occlusion & $\begin{array}{l}\text { Consider ICA and/or viability } \\
\text { assessment }\end{array}$ \\
\hline CAD-RADS N & Nondiagnostic & $\begin{array}{l}\text { Obstructive CAD cannot be } \\
\text { excluded }\end{array}$ & CAD-RADS N \\
\hline
\end{tabular}

Abbreviations: CAD, coronary artery disease; CAD-RADS, Coronary Artery Disease-Reporting and Data System; ICA, invasive coronary angiography.

Table 2 Patient characteristic and CAD-RADS

\begin{tabular}{|c|c|c|c|c|c|c|c|}
\hline Variable & $\begin{array}{l}\text { CAD-RADS } \\
0\end{array}$ & $\begin{array}{l}\text { CAD-RADS } \\
1\end{array}$ & $\begin{array}{l}\text { CAD-RADS } \\
2\end{array}$ & $\begin{array}{l}\text { CAD-RADS } \\
3\end{array}$ & $\begin{array}{l}\text { CAD-RADS } \\
4 \mathrm{a}\end{array}$ & $\begin{array}{l}\text { CAD-RADS } \\
4 \mathrm{~b}\end{array}$ & $\begin{array}{l}\text { CAD-RADS } \\
5\end{array}$ \\
\hline Patient $(\%, n)$ & $91 \%, 25$ & $83 \%, 23$ & $75 \%, 20$ & $69 \%, 19$ & $19 \%, 5$ & $13 \%, 3$ & $9 \%, 2$ \\
\hline Age (y) & $47 \pm 10$ & $51 \pm 14$ & $49 \pm 12$ & $55 \pm 9$ & $56 \pm 8$ & $51 \pm 11$ & $62 \pm 10$ \\
\hline Gender $(n)$ & 56 & 40 & 35 & 27 & 3 & 2 & 1 \\
\hline Hypertension & 30 & 20 & 43 & 50 & 13 & 8 & 5 \\
\hline Diabetes mellitus & 7 & 4 & 8 & 9 & 4 & 3 & 3 \\
\hline Smoking & 50 & 55 & 57 & 63 & 17 & 11 & 8 \\
\hline Family history & 45 & 20 & 32 & 40 & 12 & 8 & 3 \\
\hline MACE + & 1 & 3 & 3 & 7 & 4 & 3 & 4 \\
\hline MACE- & $99 \%$ & $98 \%$ & $96 \%$ & $91 \%$ & $79 \%$ & $75 \%$ & $50 \%$ \\
\hline
\end{tabular}

Abbreviations: CAD-RADS, Coronary Artery Disease-Reporting and Data System; MACE, major adverse cardiac event.

Table 3 Multivariate models were adjusted for patient age, gender, hypertension, diabetes mellitus, smoking, and family history

\begin{tabular}{|l|l|l|l|l|}
\hline & Univariable HR & $p$-Value & Multivariable HR & $p$-Value \\
\hline CAD-RADS 0 & 1.00 & Reference & 1.00 & Reference \\
\hline CAD-RADS 1 & $2.54(1.91-3.82)$ & $<0.0001$ & $2.53(1.81-3.21)$ & $<0.0001$ \\
\hline CAD-RADS 2 & $3.19(2.55-5.01)$ & $<0.0001$ & $3.18(2.35-4.42)$ & $<0.0001$ \\
\hline CAD-RADS 3 & $3.43(2.51-4.71)$ & $<0.0001$ & $3.42(2.29-5.11)$ & $<0.0001$ \\
\hline CAD-RADS 4A & $3.96(3.10-4.43)$ & $<0.0001$ & $3.91(2.54-5.06)$ & $<0.0001$ \\
\hline CAD-RADS 4B & $7.01(5.46-9.01)$ & $<0.0001$ & $6.19(4.06-7.77)$ & $<0.0001$ \\
\hline CAD-RADS 5 & $7.21(4.94-9.91)$ & $<0.0001$ & $6.29(4.94-9.14)$ & $<0.0001$ \\
\hline
\end{tabular}

Abbreviation: CAD-RADS, Coronary Artery Disease-Reporting and Data System.

CAD-RADS is sufficient evidence for invasive procedures in cases. In these cases, the expectation of MACE at follow-up has increased.

CAD-RADS scoring is important for deciding on treatment. ${ }^{4}$ But this, alone, cannot improve clinical outcomes. Clinical assessment, risk factors, and noninvasive imaging results are crucial for identifying the appropriate treatment for patients. The use of ICA has been reduced with CAD-RADS scoring. ${ }^{4}$ In literature, data similar to our study have been found in follow-up cases after CTA. MACE development in patients is classified according to the lumen stenosis observed in CTA. Pundziute et al observed MACE in 20 of 32 patients with severe stenosis in a study with 16 months mean follow-up time in 100 cases in 2007. ${ }^{10}$ Hadamitzky et al followed patients after coronary artery bypass graft with an average of 1,150 cases for a mean of 18 months in 2009, while Chow et al followed patients with an average of 2,076 cases for a mean of 16 months. ${ }^{12}$ The common characteristic of these studies is that they are short-term and CAD-RADS classification is not used. ${ }^{5,12,13}$ Xie et al evaluated 5,076 cases in 5 years. This study is the largest series study using CAD-RADS scoring. ${ }^{4}$ In this study, we have a longer follow-up period than Xie et al with our 10-year follow-up. The results of a meta-analysis in the literature data are compatible with our study. ${ }^{3}$ 


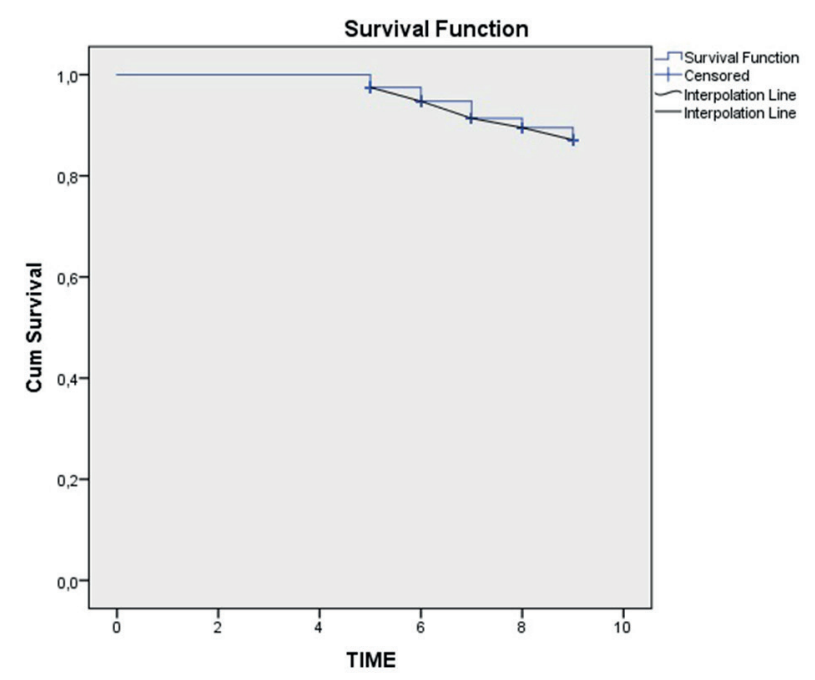

Fig. 1 Coronary Artery Disease-Reporting and Data System scoring and incidence analysis with cumulative without major adverse cardiac event in 10-year follow-up.

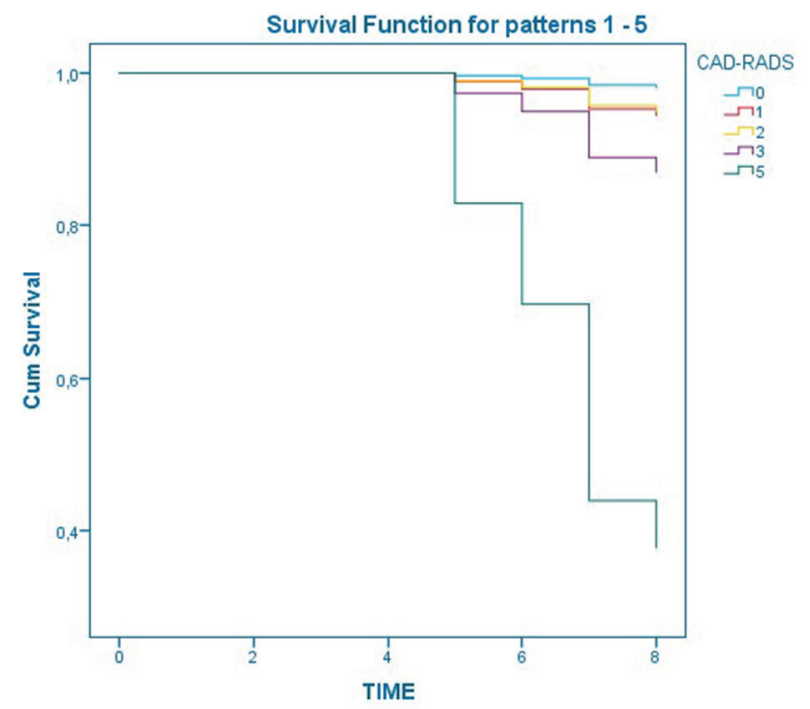

Fig. 2 Cox regression-major adverse cardiac events incidence of for Coronary Artery Disease-Reporting and Data System (CAD-RADS) scores.

Detailed risk analysis, clinical and radiological follow-up guidelines are available for diagnosis and follow-up of CAD. ${ }^{14}$ These guidelines are updated with new data. Standardization of radiological reporting with CAD-RADS has caused some changes in guidelines. ${ }^{1,13}$ Stress and perfusion examinations, invasive angiography examinations were applied to patients with higher CAD-RADS with more precise indications. Follow-up and medical treatment came to the fore in patients with low CAD-RADS scores. ${ }^{1,11}$ Clinical confidence in CAD-RADS scoring increases with studies similar to our study containing long-term clinical data. Radiological examination and survey studies have been a reliable guide in coronary patients. The high level of foresight with CAD-RADS will reduce the need for interference in many potential patients

\section{Limitations of the Study}

In this study, stent control patients and bypass follow-up patients could not be evaluated. The use of CAD-RADS scoring in control CTA patients should be investigated in a large series of patients. Taking clinical data retrospectively in the study resulted in statistics with clinical findings interpreted by multiple researchers. Case follow-ups were made from hospital records except for 15 patients. Our methods of accessing clinical data were, therefore, evaluated as limitations. No planned standard clinical examination could be performed on the patients. One of the major limitations was that the radiology reports were evaluated through the written archive and and CAD-RADS scoring was performed by reports. Another limitation was that our cases could not be evaluated in terms of graft, stent, or vulnerable plaque. Failure to reevaluate cases through PACS was a major limitation.

\section{Conclusion}

In this study, the clinical prediction of 10-year CAD-RADS scores was evaluated. As a result, in long-term follow-up, CAD-RADS scoring gave very significant results in predicting side effects related to CAD. In this study, results similar to that of the literature studies were achieved. It is necessary to evaluate CAD-RADS scoring reports as a new criterion in the follow-up of patients after CTA. This study should be extended to a prospective, multicentered study with a greater number of patients. The impact of the presence of stent, bypass, and vulnerable plaques on the survey should be evaluated.

\section{Financial Support and Sponsorship}

Nil.

\section{Conflict of Interest}

There are no conflicts of interest.

\section{Note}

This article is not under consideration elsewhere. None of the paper's contents, except for abstracts, have been previously published. The author has read and approved the manuscript. This study has not financial support. This study was one center study. There is no conflict of interest. Katip Çelebi University ethics committee secretariat application no: (2020-GOKAE-0261). $p<0.005$ was found significant.

\section{References}

1 Cury RC, Abbara S, Achenbach S, et al; Endorsed by the American College of Cardiology. CAD-RADS ${ }^{\mathrm{TM}}$ : Coronary Artery Disease - Reporting and Data System: an expert consensus document of the Society of Cardiovascular Computed Tomography (SCCT), the American College of Radiology (ACR) and the North American Society for Cardiovascular Imaging (NASCI) J Am Coll Radiol 2016;13(12 Pt A):1458-1466.e9

2 Cury RC, Abbara S, Achenbach S, et al. CAD-RADS ${ }^{\mathrm{TM}}$ Coronary Artery Disease-Reporting and Data System. An expert consensus document of the Society of Cardiovascular Computed Tomography (SCCT), the American College of Radiology (ACR) 
and the North American Society for Cardiovascular Imaging (NASCI). Endorsed by the American College of Cardiology. J Cardiovasc Comput Tomogr 2016;10:269-281

3 Abdulla J, Asferg C, Kofoed KF. Prognostic value of absence or presence of coronary artery disease determined by 64-slice computed tomography coronary angiography a systematic review and meta-analysis. Int J Cardiovasc Imaging 2011;27(3):413-420

4 Xie JX, Cury RC, Leipsic J, et al. The Coronary artery disease-reporting and data system (CAD-RADS): prognostic and clinical implications associated with standardized coronary computed tomography angiography reporting. JACC Cardiovasc Imaging 2018;11(1):78-89

5 Gaemperli O, Valenta I, Schepis T, et al. Coronary 64-slice CT angiography predicts outcome in patients with known or suspected coronary artery disease. Eur Radiol 2008;18(6): 1162-1173

6 Ostrom MP, Gopal A, Ahmadi N, et al. Mortality incidence and the severity of coronary atherosclerosis assessed by computed tomography angiography. J Am Coll Cardiol 2008; 52(16):1335-1343

7 Carrigan TP, Nair D, Schoenhagen P, et al. Prognostic utility of 64-slice computed tomography in patients with suspected but no documented coronary artery disease. Eur Heart J 2009; 30(3):362-371

8 Shaw LJ, Raggi P, Schisterman E, Berman DS, Callister TQ. Prognostic value of cardiac risk factors and coronary artery calcium screening for all-cause mortality. Radiology 2003;228(3):826-833

9 van Werkhoven JM, Schuijf JD, Gaemperli O, et al. Prognostic value of multislice computed tomography and gated single-photon emission computed tomography in patients with suspected coronary artery disease. J Am Coll Cardiol 2009;53(7):623-632

10 Pundziute G, Schuijf JD, Jukema JW, et al. Prognostic value of multislice computed tomography coronary angiography in patients with known or suspected coronary artery disease. J Am Coll Cardiol 2007;49(1):62-70

11 Nakamura S, Kitagawa K, Goto Y, et al. Prognostic value of stress dynamic computed tomography perfusion with computed tomography delayed enhancement. JACC Cardiovasc Imaging 2020;13(8):1721-1734

12 Hadamitzky M, Freissmuth B, Meyer T, et al. Prognostic value of coronary computed tomographic angiography for prediction of cardiac events in patients with suspected coronary artery disease. JACC Cardiovasc Imaging 2009;2(4):404-411

13 Chow BJ, Ahmed O, Small G, et al. Prognostic value of CT angiography in coronary bypass patients. JACC Cardiovasc Imaging 2011;4(5):496-502

14 Nam K, Hur J, Han K, et al. Prognostic value of coronary artery disease-reporting and data system (CAD-RADS) score for cardiovascular events in ischemic stroke. Atherosclerosis 2019;287:1-7 